Article

\title{
Cellular Internalization of Beta-Carotene Loaded Polyelectrolyte Multilayer Capsules by Raman Mapping
}

\author{
Loredana F. Leopold ${ }^{1}$, Oana Marișca ${ }^{2, *}$, Ioana Oprea ${ }^{1}$, Dumitrița Rugină ${ }^{3}$, Maria Suciu ${ }^{4}(\mathbb{D}$, \\ Mădălina Nistor ${ }^{1}$, Maria Tofană ${ }^{1}$, Nicolae Leopold ${ }^{2}$ (D) and Cristina Coman ${ }^{1, *(D)}$ \\ 1 Faculty of Food Science and Technology, University of Agricultural Sciences and Veterinary Medicine, Calea \\ Mănăștur 3-5, 400372 Cluj-Napoca, Romania; loredana.leopold@usamvcluj.ro (L.F.L.); \\ ioana.oprea@usamvcluj.ro (I.O.); madalina.nistor@usamvcluj.ro (M.N.); maria.tofana@usamvcluj.ro (M.T.) \\ 2 Faculty of Physics, Babeș-Bolyai University, Kogalniceanu 1, 400084 Cluj-Napoca, Romania; \\ nicolae.leopold@phys.ubbcluj.ro \\ 3 Faculty of Veterinary Medicine, University of Agricultural Sciences and Veterinary Medicine, Calea \\ Mănăștur 3-5, 400372 Cluj-Napoca, Romania; dumitrita.rugina@usamvcluj.ro \\ 4 National Institute for Research and Development of Isotopic and Molecular Technologies, Donath 67-103, \\ 400293 Cluj-Napoca, Romania; maria.suciu@itim-cj.ro \\ * Correspondence: oanamarisca@gmail.com (O.M.); cristina.coman@usamvcluj.ro (C.C.); \\ Tel.: +40-746-959-157 (C.C.)
}

Received: 18 February 2020; Accepted: 21 March 2020; Published: 25 March 2020 updates

\begin{abstract}
Raman mapping is becoming a very useful tool in investigating cells and cellular components, as well as bioactive molecules intracellularly. In this study, we have encapsulated beta-carotene using a layer-by-layer technique, as a way to enhance its stability and bioavailability. Further, we have used Raman mapping to characterize the as-obtained capsules and monitor their uptake by the human retinal epithelial D407 cells. We were able to successfully map the beta-carotene distribution inside the capsules, to localize the capsules intracellularly, and distinguish between capsules and other cellular components.
\end{abstract}

Keywords: beta-carotene; Raman mapping; polyelectrolyte multilayer; microcapsules; encapsulation

\section{Introduction}

An efficient tool to preserve bioactive compounds is microencapsulation [1]. Its multiple facets provide a reliable method to use in food and health industry for safe delivery of unstable extracts, vitamins, nutraceuticals, or drugs [2]. The beauty of microencapsulation resides in its ability to custom design the process for its desired application. For instance, one can protect the active compounds from oxygen, heat or light degradation, or it can encapsulate poor water-soluble compounds, or it can preserve its antioxidant activity, or just mask some organoleptic problems such as bad taste or odor [3].

Layer-by-layer assembly is one of the mostly used encapsulation techniques [4]. It is an intelligent system based on coating of a "sacrificial" template with electrostatic interacting polymers while adding layers in a controlled manner or other adjuvants, such as dyes, plasmonic nanoparticles, magnetic nanoparticles, thus tailoring the capsule towards its desired application. During the core formation, the bioactive compound is added, and it is passively trapped in the core pores and subsequently protected by addition of coating layers. Such a delivery system gained a high scientific interest due to its targeted delivery, ease of preparation, tunable characteristics, biodegradability, and biocompatibility [5].

Beta-carotene is one of the mostly known carotenoids with a high provitamin A activity whose low bioavailability, poor water solubility, and environment induced instability makes it a good candidate 
compound for micro-encapsulation [6]. Moreover, beta-carotene was extensively studied for its health benefits which comprise of vitamin A precursor, high antioxidant activity, and direct correlation with low incidence of heart disease and macular degeneration.

It was found that encapsulation of beta-carotene enhances its bioavailability while preserving its active form [7]. One of the most popular encapsulation techniques employed for beta-carotene is spray-drying [8]. In this technique, the wall material is very important, thus there are a multitude of studies on different wall types such as polysaccharides or natural polymers. Other encapsulation techniques use lipid-based particles and emulsions $[9,10]$.

Raman mapping/imaging is one of the characterization techniques used more and more in cellular imaging [11]. This method provides the advantage of being noninvasive, label-free, highly specific, and spatially accurate [12].

In this study, we decided to employ a layer-by-layer (LBL) technique using polyelectrolyte polymers to encapsulate beta-carotene. Beta-carotene was successfully entrapped in PSS/PAH (poly (sodium 4-styrene sulfonate) and poly (allylamine hydrochloride)) microcapsules. The intracellular fate of LBL polyelectrolyte capsules was previously investigated and it was found that such microcapsules reach the cell cytosol by endocytotic pathways [13]. The capsules formed in our study were studied through Raman spectroscopy in combination with multivariate statistical analysis. Furthermore, their uptake and stability were studied in human retinal epithelial D407 cells through Raman mapping.

\section{Results}

Prior to cell-microcapsules mapping, empty and loaded polyelectrolyte multilayer (PEM) capsules were characterized, and Raman screened to establish the distinct spectral features of the components.

\subsection{Capsules Characterization}

Post synthesis, the microcapsules were characterized by dynamic light scattering (DLS) and zeta-potential measurements to assess the capsules size, distribution, uniformity, and surface charge. A summary regarding these properties is presented in Figure 1c. These physical-chemical properties are important in order to predict the cell-microcapsule interaction.

Due to the nature of the microcapsule's synthesis process, one can choose how many layers the wall has, thus how big the capsule is, and also what type of charge the exterior polymeric layer has, positive or negative. In this particular case, we started with the negative polymer PSS and ended with the positive one PAH. The reasoning behind choosing six layers is because they would offer enough protection against beta-carotene leakage while maintaining the size of the capsules in the lower micro-range. Furthermore, the last layer is positively charged in order to promote an electrostatic interaction with the cellular membrane which is negatively charged thus enhancing the microcapsule's cellular uptake. According to the HPLC analysis, the encapsulation efficiency of beta-carotene is about $93 \%$.

According to the DLS and zeta-potential measurements, the average of three separate read-outs indicate that the beta-carotene microcapsules have a hydrodynamic diameter of approximately $1.3 \mu \mathrm{m}$ and a surface charge of $+16.8 \mathrm{mV}$ (Figure 1c). Analysis of TEM images (not shown here) indicate a size distribution of the microcapsules is in the 1-5 $\mu \mathrm{m}$ range. SEM analysis showed uniform round capsules with a finely rough surface (Figure $1 b$ ).

Figure 1a shows the Raman fingerprint of the nonloaded microcapsules, beta-carotene loaded microcapsules, and beta-carotene powder. By analyzing the beta-carotene Raman fingerprint peaks measured from the beta-carotene powder, two major peaks arise. The first one at $1155 \mathrm{~cm}^{-1}$, corresponds to $\mathrm{C}-\mathrm{C}$ stretching and the second one at $1512 \mathrm{~cm}^{-1}$ corresponds to collective $\mathrm{C}=\mathrm{C}$ stretching of the conjugated unsaturated backbone of beta-carotene. A third smaller peak at $1006 \mathrm{~cm}^{-1}$ is attributed to $\mathrm{C}-\mathrm{CH}_{3}$ stretching [14-16]. The beta-carotene loaded capsules exhibit a Raman profile very similar to the Raman spectrum of beta-carotene powder. Basically, the same three vibrations of beta-carotene are visible in the characteristic Raman spectrum of beta-carotene-loaded capsules [17]. It is noticeable that 
the polymers that make up the capsule wall do not contribute to the beta-carotene loaded capsules Raman signal, beta-carotene being the major contributor to the signal.

The Raman spectrum of nonloaded capsules was also recorded, which indicates that the characteristic peaks for the polymers PSS and PAH (the polymers found in the capsule's wall) are at $1126 \mathrm{~cm}^{-1}$ which corresponds to the C-C stretching vibration from the polymer chain, and $1597 \mathrm{~cm}^{-1}$ which is associated to the $\mathrm{C}=\mathrm{C}$ aromatic ring quadrant stretching from the PSS polymer [18-20].

(a)

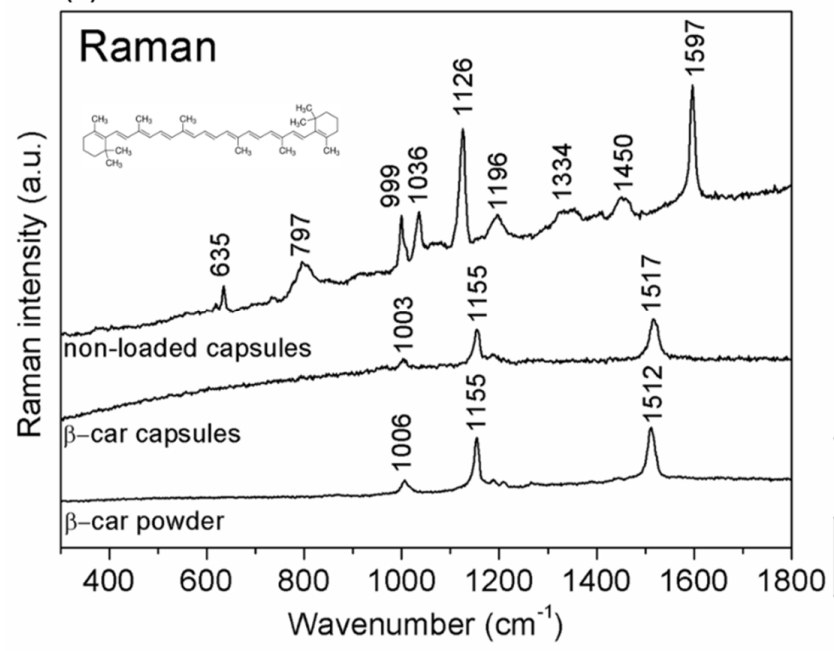

(b)

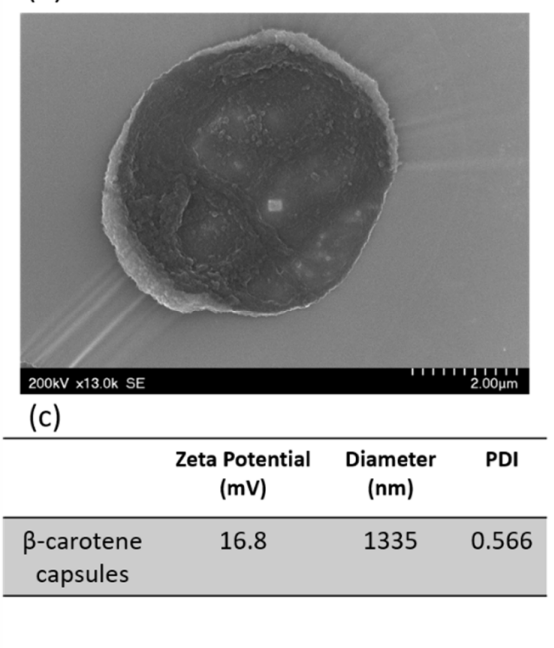

Figure 1. (a) From top to bottom, Raman spectra of empty polyelectrolyte multilayer (PEM) capsules, beta-carotene PEM capsules, and beta-carotene powder; (b) SEM image of one PEM capsule; (c) zeta potential values and hydrodynamic diameter of the beta-carotene PEM capsules.

After this basic Raman analysis of the microcapsules, the focus shifted further analyzing a multitude of Raman spectra collected at different spatial points in the sample and to transform these into score plots using relevant principal component analysis (PCA). PCA results in several significant principal components (PCs) which carry appropriate information on the analyzed samples [21]. PCA of the recorded Raman spectra provided false color score plots (maps) and their corresponding loading plots. Blue color is arbitrarily attributed to most negative values in the loading plots, while red corresponds to most positive values in the loadings. The maps are obtained by integration over the whole wavenumber range recorded.

\subsection{Raman Mapping}

Figure 2 shows the Raman map of nonloaded PEM capsules and the corresponding loading vector associated with the first PC. The loading vector of PC1 contains the characteristic Raman bands of PSS/PAH, at 1126 and $1597 \mathrm{~cm}^{-1}$. The PCA score and loading plots corresponding to the first ten PCs are represented in Figure S1.

The color map in the score plot depicts low intensities of the loading vector in each pixel with blue and high intensities of the loading vector with red. Accordingly, the pixels that contain the capsules show a high intensity of 1126 and $1597 \mathrm{~cm}^{-1}$ and consequently are assigned the red color, while the background shows practically no Raman signal and is assigned blue color. 

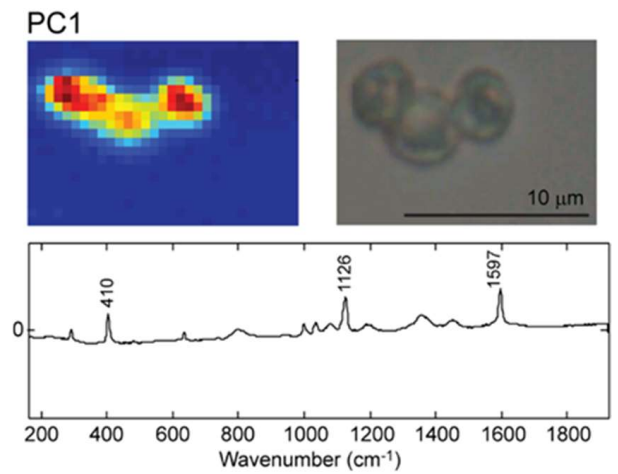

Figure 2. Top left: The false color Raman map of nonloaded microcapsules. Top Right: Optical microscopy image of nonloaded microcapsules. Bottom: The corresponding loading plot of the relevant principal component principal component 1 (PC1).

When comparing the microscopy image of the capsules, it can be observed that the Raman map replicates the image and allows easily discrimination of the three capsules analyzed. This is proof that the Raman mapping can be used as a tool to selectively image a 3D structure based on the Raman fingerprint of the structure.

A similar analysis was performed on beta-carotene loaded PEMs, shown in Figure 3. For the beta-carotene loaded PEM capsules, the loading vector corresponding to PC1 shows the characteristic vibrations from beta-carotene. Therefore, the intensity of the PC1 loading vector in each pixel of the mapped sample forms the corresponding score plot. The PCA score and loading plots corresponding to the first ten PCs are shown in Figure S2.

As before, red color signifies areas in the sample with a high Raman intensity of beta-carotene, whereas blue color is assigned to areas in the mapped sample with no Raman bands from betacarotene. Noticeably, the zones abundant in beta-carotene are in the center of the capsules, which indicates that beta-carotene is successfully entrapped in the capsules and no leaks are detected with Raman spectroscopy.

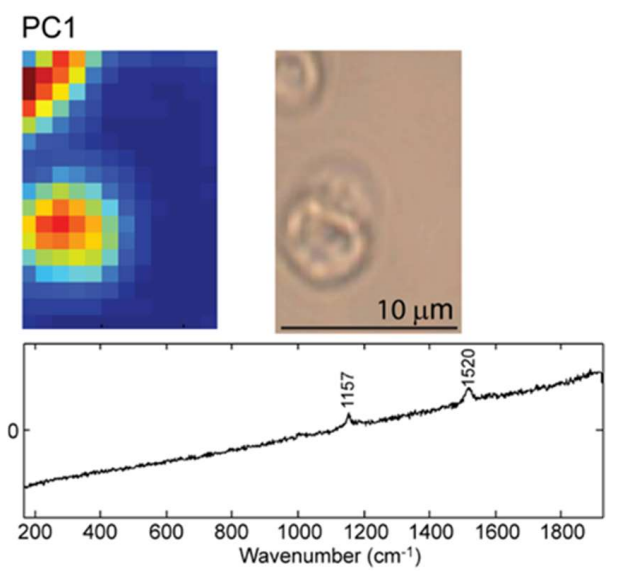

Figure 3. Top left: Raman map of beta-carotene loaded microcapsules. Top right: Microscopy image of beta-carotene microcapsules. Bottom: The corresponding loading plot of the relevant principal component PC1.

The first PC of the Raman spectra PCA provides a Raman map with very good spatial resolution, enabling the identification of the beta-carotene loaded PEM capsules. The corresponding PC1 loading plot shows the two beta-carotene marker bands at 1157 and $1520 \mathrm{~cm}^{-1}$, as well as the beta-carotene typical fluorescent background. 
Before any in vitro Raman mapping was performed, there was a need to assure that the PEM capsules are internalized by the D407 cells. The validation was obtained by TEM imaging of D407 cells. As it can be seen in Figure 4 in the TEM micrograph, three microcapsules are internalized in the D407 cell cytoplasm.

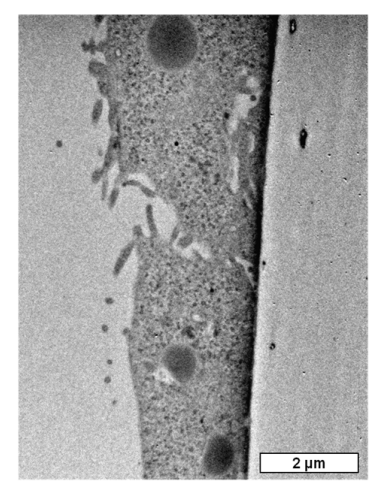

Figure 4. TEM micrograph of a D407 cell, after exposure to PEM microcapsules, showing three internalized microcapsules.

The next step further in validating the Raman mapping specificity is to evaluate the ability to discriminate the compounds of interest in an in vitro setting. The D407 cells were exposed to empty PEMs for $24 \mathrm{~h}$ and then they were imaged and analyzed via Raman spectroscopy. Figure 5 presents a collection of maps corresponding to the most significant PCs obtained by PCA of the Raman spectra registered.
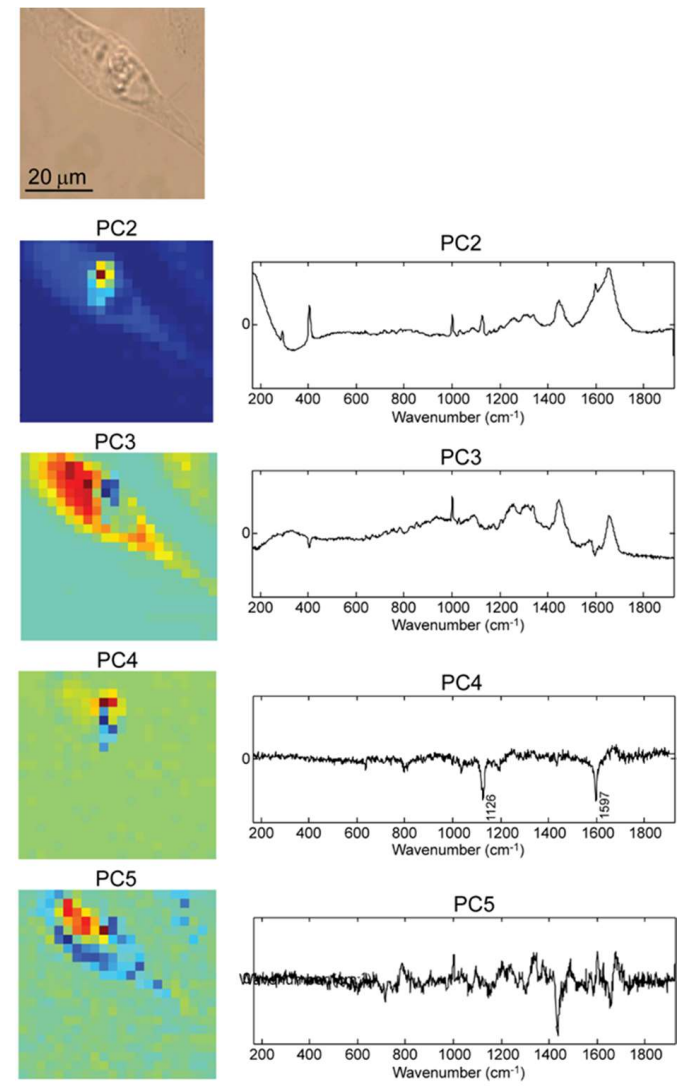

Figure 5. From top to bottom: Optical microscopy image of a D407 cell transfected with empty PEM capsules; followed by the Raman score plot (map) and the corresponding loading plot of relevant principal component PC2, PC3, PC4, and PC5. 
The PC2 loading plot in Figure 5 shows positive bands due to $\mathrm{MgF}_{2}$ (used as substrate for the cells) at $406 \mathrm{~cm}^{-1}$, and a superposition of bands due to the polymer $\left(1126,1598 \mathrm{~cm}^{-1}\right)$ and typical cell Raman bands, the most evident being at 1001,1446,1654 cm${ }^{-1}$. The band at $1001 \mathrm{~cm}^{-1}$ is assigned to aromatic ring stretching of phenylalanine amino acid from proteins, the $1446 \mathrm{~cm}^{-1}$ vibration represents the $\mathrm{C}-\mathrm{H}$ bending of lipids, and $1654 \mathrm{~cm}^{-1}$ contains mixed contribution from amide I band of proteins and water [22-24]. The PC2 score plot depicts the internalized capsule and the shape of the cell.

However, the cell shape can be observed more clearly in the score plot of PC3, the loading of PC3 showing typical cell Raman bands. The capsule shape can be recognized also in the score plot of PC3. The negatively correlated $\mathrm{MgF}_{2}$ Raman band with the cell Raman bands generates a dark blue region in the score plot, in the close vicinity of capsule.

The shape in PC4 score plot is attributed to the capsule, as also indicated by the characteristic bands in the loading plot. The negative peaks in PC4 correlate with the PSS/PAH signature peaks corresponding to the $C-C$ stretching vibrations at 1126 and $1597 \mathrm{~cm}^{-1}$ vibration associated with the aromatic ring quadrant [20]. Thus, PC4 map provides evidence that the PEM capsules are internalized in the cell and they can be easily identified among other cell structures.

Cellular components can be recognized in the score plot of PC5. Typical cellular bands of phenylalanine, lipids, amide I band of proteins are present at 1003, 1446, and $1654 \mathrm{~cm}^{-1}$, respectively, but also the $788 \mathrm{~cm}^{-1}$ positive band, which is assigned to the $\mathrm{PO}_{4}$ vibration of DNA (O-P-O stretching), the high DNA content being typical for the cell nucleus [24] shown in red color in the score plot. The negative peak at $1446 \mathrm{~cm}^{-1}$ in PC5 score plot can be assigned to C-H bending vibration of lipids; depicting these cytoplasmic components in blue color, since lipid synthesis is mainly located in the endoplasmic reticulum [23]. The spectral fingerprint representing the DNA is negatively correlated with the one representing the $\mathrm{C}-\mathrm{H}$ vibration, so nucleus is shown in red and the cytoplasm in blue. The PCA score and loading plots corresponding to the first ten PCs are shown in Figure S3.

Next, beta-carotene PEM capsules were transfected in the D407 cells and a representative Raman map is depicted in Figure 6.
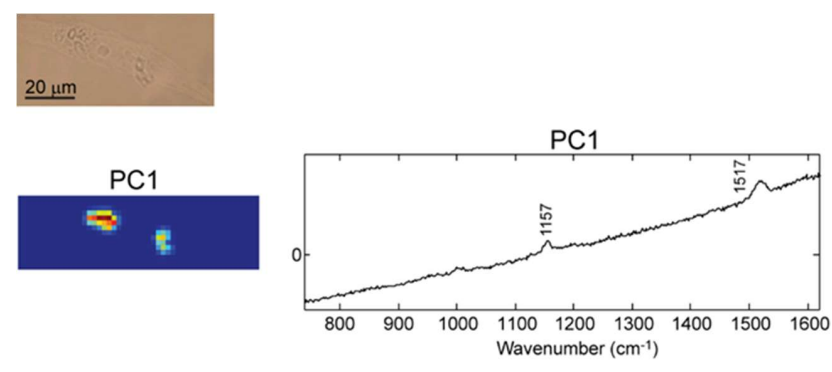

PC3
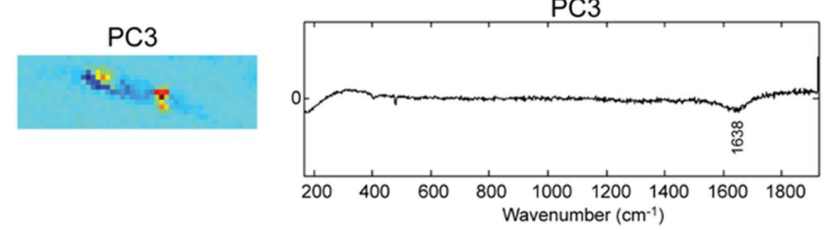

PC4
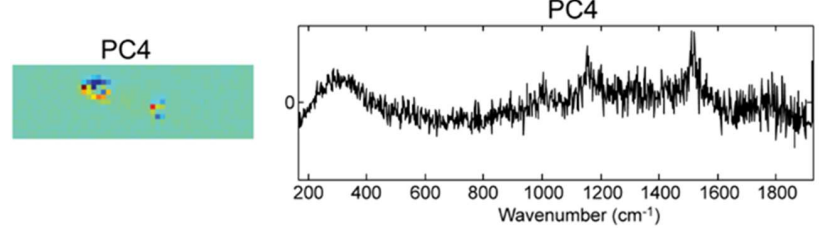

Figure 6. From top to bottom: Optical microscopy image of a D407 cell transfected with beta-carotene loaded PEM capsules followed by the Raman score plots (maps) and the corresponding loading plots of relevant principal components PC1, PC3, and PC4. 
In order to avoid beta-carotene degradation upon exposure to laser light, very low laser power $(\mathrm{mW})$ was used when imaging cells exposed to this type of capsule.

As shown in Figure 6, PC1 comprises the highest variability of the spectra, the loading plot showing a typical fluorescence background emitted by beta-carotene when excited with $532 \mathrm{~nm}$ laser. The Raman bands assigned to $C-C\left(1157 \mathrm{~cm}^{-1}\right)$ and $C=C\left(1517 \mathrm{~cm}^{-1}\right)$ stretching vibrations of beta-carotene backbone can be observed as well on the fluorescent background. Thus, the two structures observed in the PC1 score plot of Figure 6 are assigned to the beta-carotene loaded capsules.

The score plot in PC3 displays in blue color the cell shape, the beta-carotene capsules being observed in yellow-red colors. The negative bands in the loading plot, below 300 and at $1640 \mathrm{~cm}^{-1}$, are assigned to typical water vibrations. The red yellow-red color of the beta-carotene capsules is mainly due to the positive values in the spectral part higher than $1700 \mathrm{~cm}^{-1}$, due to the fluorescence pattern of beta-carotene.

The loading plot of PC4 shows clearly beta-carotene bands at 1153 and $1516 \mathrm{~cm}^{-1}$ in good concordance with the two beta-carotene capsules shown in the score plot of PC4. The PCA score and loading plots corresponding to the first ten PCs are shown in Figure S4.

So, the Raman imaging technique was able to discriminate the major cellular components, the PEMs, and the beta-carotene PEM capsules.

\section{Materials and Methods}

\subsection{Materials and Reagents}

The poly (allylamine hydrochloride), $\mathrm{PAH}, \mathrm{Mw} 900,000 \mathrm{~g} / \mathrm{mol}$, and poly (sodium 4-styrene sulfonate), PSS, Mw 70,000 g/mol were purchased from Aldrich, dextran sulfate sodium salt MW 40,000 g/mol was purchased from AppliChem, $\mathrm{CaCl}_{2}$, and $\mathrm{Na}_{2} \mathrm{CO}_{3}$ from VWR, beta-carotene was supplied from Cayman Chemical Company (Ann Arbor, MI, USA), DMEM from Gibco.

\subsection{Synthesis of Polyelectrolyte Multilayer Capsules}

The PEM capsules were built around $\mathrm{CaCO}_{3}$ core, following a previously reported protocol [25] (with some minor changes). The core serves as template only and, at the end, after all polymer layers were deposited, it was solubilized with EDTA. For the formation of the $\mathrm{CaCO}_{3}$ template core, equal amounts $(6 \mathrm{~mL})$ of $0.33 \mathrm{M} \mathrm{CaCl}_{2}$ and $\mathrm{Na}_{2} \mathrm{CO}_{3}$ were mixed under stirring at room temperature. Subsequently, $7.5 \mathrm{~mL}$ dextran sulfate $5 \mathrm{mg} / \mathrm{mL}$ was added (dextran is efficient in solubilizing the $\mathrm{CaCO}_{3}$ core at the final step). The polyelectrolyte layers were built around the $\mathrm{CaCO}_{3}$ cores using alternating layers of positively charged poly (allylamine hydrochloride), $\mathrm{PAH}$, and negatively charged poly (4-styrene sulfonate), PSS, $2 \mathrm{~g} / \mathrm{L}, 10 \mathrm{~mL}$, prepared in $0.5 \mathrm{M} \mathrm{NaCl}$. Three washing cycles with water (centrifugation, removing supernatant, addition of water) were carried out after the formation of each polymer layer. In total, six polymer layers were formed, followed by the dissolution of the $\mathrm{CaCO}_{3}$ core using $10 \mathrm{~mL}$ EDTA solution $0.2 \mathrm{M}, \mathrm{pH}$ 5.5. Eight washing steps in water were performed after core dissolution, by centrifugation at $120 \mathrm{~g}, 8 \mathrm{~min}$. The final structure of the obtained PEM capsules is PSS/PAH/PSS/PAH/PSS/PAH. To obtain the beta-carotene loaded microcapsules, first a stock solution of beta-carotene $(5 \mathrm{mg} / \mathrm{mL}$ in tetrahydrofuran) was prepared. Desired amounts of solubilized beta-carotene were mixed with the dextran solution and added after the core formation step.

\subsection{Beta-Carotene Encapsulation Efficiency}

The beta-carotene encapsulation efficiency was quantified using an Agilent HPLC instrument coupled with a DAD detector (Agilent Tehnologies, Santa Clara, CA, USA), using a reversed phase EC 250/4.6 Nucleodur 300-5 C-18 column $(250 \times 4.6 \mathrm{~mm}), 5 \mu \mathrm{m}$ (Macherey-Nagel, Germany). The mobile phase consisted of mixtures of acetonitrile: Water $(9: 1, v / v)$ with $0.25 \%$ triethylamine $(\mathrm{A})$ and ethyl acetate with $0.25 \%$ triethylamine (B). The gradient started with $90 \% \mathrm{~A}$ at $0 \mathrm{~min}$ to $50 \% \mathrm{~A}$ at $10 \mathrm{~min}$. The percentage of A decreased from $50 \%$ at $10 \mathrm{~min}$ to $10 \%$ A at $20 \mathrm{~min}$. The flow rate was $1 \mathrm{~mL} / \mathrm{min}$ and 
the chromatogram was recorded at $450 \mathrm{~nm}$ [26]. The HPLC peaks were identified and quantified based on a calibration curve registered using freshly prepared beta-carotene standard solutions (Cayman Chemical, Ann Harbor, MI, USA). In short, sampling was carried out after each single step in the PEM microcapsules synthesis (after core formation, after adding each polymer, after every single washing step, after adding EDTA). This resulted in a total of 30 samples. From each of these aqueous samples, beta-carotene was extracted and quantified.

In short, $1 \mathrm{~mL}$ of ethyl acetate was used to extract beta-carotene from each aqueous sample. After extraction, the organic phase was collected, the solvent was evaporated, and the dried samples were kept at freezing until the next day, when the beta-carotene was again solubilized in ethyl acetate and $20 \mu \mathrm{L}$ of the solution was injected in the HPLC column. The solvent evaporation was a necessary step since we have observed partial degradation of beta-carotene upon overnight storage if the solvent was present in the samples. Taking into account the beta-carotene instability in atmospheric conditions, the stock solution was also quantified by HPLC to have a correct estimation of the total amount that could be encapsulated. The nonencapsulated beta-carotene was quantified by summing up beta-carotene that resulted from each washing step. The difference between the initial beta-carotene amount and the amount lost during the synthesis represents the encapsulated beta-carotene. The nonencapsulated beta-carotene was calculated from the calibration curve with the equation $y=0.3961 x+6.6094, R^{2}=0.9918$. For the stock solution, the equation $y=1.6793 x-3280.7$, $\mathrm{R}^{2}=0.995$ was used.

\subsection{Cell Culture and Treatment}

The human retinal epithelial D407 cell line was kindly donated by Prof. em. Dr.Dr.h.c. Horst A. Diehl, University of Bremen. The cells were cultured in Dulbecco's Modified Eagle Medium supplemented with $10 \%$ fetal bovine serum, $1 \mathrm{mM}$ sodium pyruvate, $100 \mathrm{U} / \mathrm{mL}$ penicillin, $100 \mathrm{mg} / \mathrm{mL}$ streptomycin, and $2.5 \mathrm{mg} / \mathrm{mL}$ amphotericin $\mathrm{B}$. The cells were incubated at $37^{\circ} \mathrm{C}$, in $5 \% \mathrm{CO}_{2}$ atmosphere, and $95 \%$ relative humidity. For the Raman mapping experiments the cells were grown on $35 \mathrm{~mm}$ sterile Petri dishes and seeded on $\mathrm{MgF}_{2}$ plates, as detailed in the section below.

\subsection{Methods}

\subsubsection{Raman Spectroscopy and Mapping}

The Raman spectra were recorded with a Renishaw inVia Reflex Raman Spectrometer (Renishaw, New Mills, UK), equipped with an upright Leica microscope, an 1800 lines/mm grating, a CCD detector, and an automated step motorize stage to raster scan the sample area during the mapping experiments. The spectra were recorded using the $532 \mathrm{~nm}$ excitation laser line. The spectral resolution was $4 \mathrm{~cm}^{-1}$. Prior to spectral acquisitions, the wavelength was calibrated using an internal Si standard. The laser power during measurement was adjusted to either 120,6, or $1 \mathrm{~mW}$. Laser power of $120 \mathrm{~mW}$ was used for spectral acquisitions on samples containing empty PEM capsules, while for beta-carotene loaded capsules, adjustment of laser power to 6 or $1 \mathrm{~mW}$ was needed in order to avoid degradation of the beta-carotene molecule, which is heat and light sensitive. Spectra preprocessing consisted of cosmic ray removal.

$\mathrm{MgF}_{2}$ plates were used as solid support for the samples. $\mathrm{MgF}_{2}$ is a suitable support for Raman analysis, due to its extreme low fluorescent background. To record the Raman spectra on the PEM microcapsules, a drop of aqueous dispersion containing the capsules was deposited onto $\mathrm{MgF}_{2}$ plates and allowed to dry at room temperature. The samples were further analyzed under the Olympus 100X objective. The Raman maps were recorded by raster scanning the desired sample areas with 1 acquisition and $2 \mathrm{~s}$ exposure in each point. For imaging the nonloaded capsules, a $0.5 \mu \mathrm{m}$ step size and $60 \mathrm{~mW}$ laser power were used, and for the beta-carotene loaded capsules $1 \mu \mathrm{m}$ step size and $6 \mathrm{~mW}$ laser power were chosen. All data processing was carried out using custom-built MATLAB 
R2019b (The MathWorks Inc., Natick, MA, USA) functions. Cosmic ray spikes were removed from each spectrum before processing. For the identification of various cell components, PCA was performed.

To record Raman spectra on D407 cells upon exposure to different formulations of PEM capsules, the cells were grown for $24 \mathrm{~h}$ on sterile Petri dishes of $35 \mathrm{~mm}$ diameter $\left(1.2 \times 10^{5}\right.$ cells seeded). The day after, the growth medium was replaced with PEM capsules diluted in the cell culture medium at a concentration of 10 capsules/cell, for another $24 \mathrm{~h}$. Further, D407 cells were washed three times with phosphate buffer saline (PBS), detached from the Petri dishes using trypsin, centrifuged, and reseeded in Petri dishes with $\mathrm{MgF}_{2}$ plates inside. The PEM capsules treated cells were allowed to attach to the $\mathrm{MgF}_{2}$ plates for $24 \mathrm{~h}$, followed by three times washing with PBS and cell fixation onto the plates. Cell fixation was carried out with $4 \%$ paraformaldehyde, at room temperature, for $20 \mathrm{~min}$. After immobilization, the samples were again washed and stored in PBS buffer. The D407 cells exposed to PEM microcapsules were analyzed under the Olympus 60x water immersion objective. The maps were recorded with $2 \mu \mathrm{m}$ step size, $1 \mathrm{~s}$ exposure time and 1 acquisition at each measuring point, and a laser power of $120 \mathrm{~mW}$ (samples exposed to nonloaded PEM capsules) or $6 \mathrm{~mW}$ (samples exposed to beta-carotene loaded PEM capsules).

\subsubsection{Multivariate Data Analysis}

Data processing, including PCA and cosmic ray removal was carried out using custom-built MATLAB functions (MathWorks, Natick, MA, USA). PCA is a statistical method which reduces the dimensionality of the data by transforming the coordinate system of the data (in this case all Raman spectra from each pixel) into a coordinate system with less dimensions, while keeping as much of the variance of the data set. Therefore, the output of PCA is a set of spectra resembling plots, termed loading vectors, which correspond to the new coordinate system of the data set, and corresponding point-by-point mappings, termed score plots. Physically, the loading vectors represent the Raman bands that explain best the variance in the data set, i.e., how the Raman spectra of the sample in each pixel changes. Since loading vectors do not represent physically obtainable spectra but must be regarded as the coordinate system of the data, the interpretation of loading vectors must consider both positive and negative peaks. The score plot depicts visually the spatial correlation of the loading vector with the mapped sample. The biochemical structures displayed in the score plots can be assigned by identifying the biochemical information provided by the loading vector in the corresponding pixels [27].

Prior to PCA, cosmic ray removal was performed, which eliminates any artifact bands from the Raman spectrum. Since the PCA separates any redundant information from the Raman spectra (analogous to deconvolution), baseline removal was not necessary, since it appears only in one loading vector (due to the loading vectors being orthogonal with each other).

\subsubsection{Physical Characterization of PEM Capsules}

The hydrodynamic diameter and surface charge of the beta-carotene loaded capsules were assessed using a Malvern Zetasizer Nano ZS equipped with a $4 \mathrm{~mW}$ He-Ne laser operating at $633 \mathrm{~nm}$ and an avalanche photodiode detector (Malvern Instruments, Worcestershire, UK). Zeta potential was measured by laser Doppler electrophoresis and the hydrodynamic diameter of the particles was measured by DLS. The measurements were carried out at $25^{\circ} \mathrm{C}$. Scanning electron microscopy (SEM) analysis of capsules were done on Hitachi SU8230 microscope (Hitachi, Krefeld, Germany) at $200 \mathrm{kV}$ and $8 \mathrm{~mm}$ working distance.

The TEM experiments were carried out using a JEOL model JEM1010 (Jeol, Peabody, MA, USA) instrument operating at $80 \mathrm{kV}$ accelerating voltage and equipped with a MegaViewIII CCD camera. For the TEM experiments, D407 cells were seeded for $24 \mathrm{~h}$ on Falcon cell culture inserts $(0.4 \mu \mathrm{m})$ (Becton Dickinson Labware, Bedford, MA, USA), $\left(8 \times 10^{3}\right.$ cells/cell). The cells were afterwards incubated for another $24 \mathrm{~h}$ with capsules (10 capsules/well). The culture medium was then removed, and the cells were washed three times with PBS and prefixed for $1 \mathrm{~h}$ with glutaraldehyde (2.5\% in PBS). Next, three 
steps of rinsing with PBS and cells were post-fixed for $1 \mathrm{~h}$ in osmium tetroxide (2\% in PBS). Dehydration was carried out in HPLC grade acetone in distilled water dilutions, followed by embedding with Epon resin. The resin was polymerized at $60{ }^{\circ} \mathrm{C}$ for $48 \mathrm{~h}$, then cooled for $12 \mathrm{~h}$. Ultrathin sections of about $70 \mathrm{~nm}$, obtained on a diamond knife (Diatome) with Leica UC6 ultramicrotome (Leica Microsystems, Wetzlar, Germany) were post-stained with lead citrate and uranyl acetate. Sections collected on 200 mesh $\mathrm{Cu}$ grids were further examined.

\section{Conclusions}

In this study, we have obtained two formulations of polyelectrolyte multilayer capsules: Nonloaded formulations and microcapsules loaded with beta-carotene. Raman mapping was successfully used as a tool to investigate the polyelectrolyte multilayer capsules alone and in vitro, after uptake in D407 cells. Regarding the as-obtained microcapsule formulations, it was possible to distinguish between beta-carotene loaded vs. nonloaded capsules and to effectively map the beta-carotene distribution inside the microcapsules core. Moreover, after exposing the D407 cells to microcapsules, by analyzing Raman specific fingerprints of the cells and capsules, it was possible to localize the capsules intracellular information also confirmed by TEM.

Supplementary Materials: The following are available online: Figure S1. Score plot and loading plot of the first ten PCs of the PCA of the Raman map presented in Figure 2, and graphical representation of the explained variance of the first ten PCs; Figure S2. Score plot and loading plot of the first ten PCs of the PCA of the Raman map presented in Figure 3, and graphical representation of the explained variance of the first ten PCs; Figure S3. Score plot and loading plot of the first ten PCs of the PCA of the Raman map presented in Figure 5, and graphical representation of the explained variance of the first ten PCs.; Figure S4. Score plot and loading plot of the first ten PCs of the PCA of the Raman map presented in Figure 6, and graphical representation of the explained variance of the first ten PCs; Figure S5. Raman map of non-loaded microcapsules and the corresponding loading plot of the relevant principal component PC1; Figure S6. Raman map of beta-carotene loaded microcapsules and the corresponding loading plot of the relevant principal component PC1; Figure S7. Raman maps of a D407 cell transfected with non-loaded PEM capsules followed by the optical microscopy image of the cell and the corresponding loading plots of relevant principal component PC2, PC3, PC4, and PC5; Figure S8. Optical microscopy image of one D407 cell exposed to non-loaded PEM capsules, corresponding Raman maps of the PC1, PC2, PC3 and PC4 components, and the corresponding loading plots of relevant principal component PC1, PC2, PC3, and PC4; Figure S9. Raman score plots (maps) and corresponding loading plots of relevant principal components PC1 and PC3 (bottom) of a D407 cell transfected with beta-carotene loaded PEM capsules., and optical microscopy image of the analyzed cell; Figure S10. Raman score plots (maps) of a D407 cell exposed to beta-carotene loaded PEM capsules, followed by the optical microscopy image of the cell on the right, and the corresponding loading plots of relevant principal components PC1 and PC3.

Author Contributions: C.C. conceived and designed the experiments and was involved in the Raman spectroscopy measurements and manuscript writing; L.F.L. was involved in the Raman spectroscopy and mapping experiments and cell cultures preparation for Raman experiments; O.M. wrote the first draft of the manuscript and performed the first synthesis protocol of the nonloaded LBL capsules; I.O. was actively involved in the synthesis of the LBL capsules; D.R. was in charge of the cell cultures (culturing cells, cell fixation, and preparation for TEM); M.S. performed the electron microscopy analysis; M.T. provided materials and reagents and aided in interpreting the results; M.N. performed the experiments for the determination of the encapsulation yield; N.L. provided scientific support and was involved in the statistical analysis and interpretation of the Raman maps. All authors have read and agreed to the published version of the manuscript.

Funding: This work was supported by a grant from the Ministry of Research and Innovation, CNCS - UEFISCDI, project number PN-III-P1-1.1-TE-2016-1907, within PNCDI III. The publication was supported by funds from the National Research Development Project to Finance Excellence, (PFE)-37/2018-2020, granted by the Romanian Ministry of Research and Innovation.

Conflicts of Interest: The authors declare no conflict of interest.

\section{References}

1. De Koker, S.; Hoogenboom, R.; de Geest, B.G. Polymeric multilayer capsules for drug delivery. Chem. Soc. Rev. 2012, 41, 2867-2884. [CrossRef] [PubMed]

2. Dos Santos, P.P.; Flôres, S.H.; de Oliveira Rios, A.; Chisté, R.C. Biodegradable polymers as wall materials to the synthesis of bioactive compound nanocapsules. Trends Food Sci. Technol. 2016, 53, 23-33. [CrossRef] 
3. Lee, W.L.; Widjaja, E.; Loo, S.C.J. Designing drug-loaded multi-layered polymeric microparticles. J. Mater. Sci. Mater. Med. 2012, 23, 81-88. [CrossRef]

4. Yang, X.; Han, X.; Zhu, Y. (PAH/PSS) 5 microcapsules templated on silica core: Encapsulation of anticancer drug DOX and controlled release study. Colloids Surf. A 2005, 264, 49-54. [CrossRef]

5. Ariga, K.; Hill, J.P.; Ji, Q. Layer-by-layer assembly as a versatile bottom-up nanofabrication technique for exploratory research and realistic application. PCCP 2007, 9, 2319-2340. [CrossRef] [PubMed]

6. Donhowe, E.G.; Kong, F. Beta-carotene: Digestion, Microencapsulation, and In Vitro Bioavailability. Food Bioorocess Techmol. 2014, 7, 338-354. [CrossRef]

7. Singh, B.B.; Shakil, N.A.; Kumar, J.; Walia, S.; Kar, A. Development of slow release formulations of $\beta$-carotene employing amphiphilic polymers and their release kinetics study in water and different $\mathrm{pH}$ conditions. J. Food Sci. Technol. 2015, 52, 8068-8076. [CrossRef]

8. Lin, Q.; Liang, R.; Williams, P.A.; Zhong, F. Factors affecting the bioaccessibility of $\beta$-carotene in lipid-based microcapsules: Digestive conditions, the composition, structure and physical state of microcapsules. Food Hydrocoll. 2018, 77, 187-203. [CrossRef]

9. Mihalcea, L.; Turturică, M.; Ghinea, I.O.; Barbu, V.; Ioniţă, E.; Cotârleț, M.; Stănciuc, N. Encapsulation of carotenoids from sea buckthorn extracted by $\mathrm{CO}_{2}$ supercritical fluids method within whey proteins isolates matrices. Innov. Food Sci. Emerg. 2017, 42, 120-129. [CrossRef]

10. Tan, C.; Xue, J.; Abbas, S.; Feng, B.; Zhang, X.; Xia, S. Liposome as a delivery system for carotenoids: Comparative antioxidant activity of carotenoids as measured by ferric reducing antioxidant power, DPPH assay and lipid peroxidation. J. Agric. Food. Chem. 2014, 62, 6726-6735. [CrossRef]

11. Brauchle, E.; Schenke-Layland, K. Raman spectroscopy in biomedicine-non-invasive in vitro analysis of cells and extracellular matrix components in tissues. Biotechnol. J. 2013, 8, 288-297. [CrossRef] [PubMed]

12. Krafft, C.; Knetschke, T.; Funk, R.H.W.; Salzer, R. Identification of organelles and vesicles in single cells by Raman microspectroscopic mapping. Vib. Spectrosc. 2005, 38, 85-93. [CrossRef]

13. Kastl, L.; Sasse, D.; Wulf, V.; Hartmann, R.; Mircheski, J.; Ranke, C.; Carregal-Romero, S.; Martinez-Lopez, J.A.; Fernandez-Chacon, R.; Parak, W.J.; et al. Multiple Internalization Pathways of Polyelectrolyte Multilayer Capsules into Mammalian Cells. ACS Nano 2013, 7, 6605-6618. [CrossRef] [PubMed]

14. De Oliveira, V.E.; Castro, H.V.; Edwards, H.G.M.; de Oliveiraa, L.F.C. Carotenes and carotenoids in natural biological samples: A Raman spectroscopic analysis. J. Raman Spectrosc. 2010, 41, 642-650. [CrossRef]

15. Thomas, D.B.; McGoverin, C.M. The analytical niche for Raman spectroscopy in biological pigment research. Spectrosc. Eur. 2016, 28, 6-9.

16. Pilát, Z.; Bernatová, S.; Ježek, J.; Šerý, M.; Samek, O.; Zemánek, P.; Nedbal, L.; Trtílek, M. Raman microspectroscopy of algal lipid bodies: $\beta$-carotene quantification. J. Appl. Phycol. 2012, 24, 541-546. [CrossRef]

17. Tong, W.; Dong, W.; Gao, C.; Möhwald, H. Charge-controlled permeability of polyelectrolyte microcapsules. J. Phys. Chem. B 2005, 109, 13159-13165. [CrossRef]

18. Edwards, H.G.M.; Brown, D.R.; Dale, J.A.; Plant, S. Raman spectroscopy of sulfonated polystyrene resins. Vib. Spectrosc. 2000, 24, 213-224. [CrossRef]

19. Wang, F.; Feng, J.; Tong, W.; Gao, C. A facile pathway to fabricate microcapsules by in situ polyelectrolyte coacervation on poly(styrene sulfonate)-doped CaCO3 particles. J. Mater. Chem. 2007, 17, 670-676. [CrossRef]

20. De, R.; Lee, H.; Das, B. Exploring the interactions in binary mixtures of polyelectrolytes: Influence of mixture composition, concentration, and temperature on counterion condensation. J. Mol. Liq. 2018, 251, 94-99. [CrossRef]

21. Maslova, O.A.; Guimbretière, G.; Ammar, M.R.; Desgranges, L.; Jégou, C.; Canizarès, A.; Simon, P. Raman imaging and principal component analysis-based data processing on uranium oxide ceramics. Mater. Charact. 2017, 129, 260-269. [CrossRef]

22. Notingher, I.; Verrier, S.; Romanska, H.; Bishop, A.E.; Polak, J.M.; Hench, L.L. In situ characterisation of living cells by Raman spectroscopy. Spectroscopy 2002, 16, 43-51. [CrossRef]

23. Leopold, L.F.; Coman, C.; Todor, T.S.; Szabo, L.; Sorițău, O.; Virag, P.; Mihu, C.M.; Moisoiu, V.; Leopold, N. Raman Imaging of Dental Follicle Mesenchymal Stem Cells. Studia UBB Phys. 2015, 60, 69-76.

24. Notingher, I.; Hench, L.L. Raman microspectroscopy: A noninvasive tool for studies of individual living cells in vitro. Expert Rev. Med. Devices 2006, 3, 215-234. [CrossRef] [PubMed] 
25. Del Mercato, L.L.; Gonzalez, E.; Abbasi, A.Z.; Parak, W.J.; Puntes, V. Synthesis and evaluation of gold nanoparticle-modified polyelectrolyte capsules under microwave irradiation for remotely controlled release for cargo. J. Mater. Chem. 2011, 21, 11468-11471. [CrossRef]

26. Pintea, A. HPLC analysis of carotenoids in four varieties of Calendula officinalis L. flowers. Acta Biol. Szeged. 2003, 47, 37-40.

27. Bonnier, F.; Byrne, H.J. Understanding the molecular information contained in principal component analysis of vibrational spectra of biological systems. Analyst 2012, 137, 322-332. [CrossRef]

Sample Availability: Not available.

(C) 2020 by the authors. Licensee MDPI, Basel, Switzerland. This article is an open access article distributed under the terms and conditions of the Creative Commons Attribution (CC BY) license (http://creativecommons.org/licenses/by/4.0/). 